\title{
PHÁT TRIỂN HỆ WEBGIS CHO PHÉP KHAI THÁC DŨ LIỆ MẠNG LƯỚI THOÁT NƯỚC THÔNG QUA INTERNET
}

\author{
TRÀ̀N TRỌNG ĐỨC \\ Truòng Đại học Bách Khoa - ĐHQG-HCM
}

\section{Tóm tắt:}

Để quản lý thông tin về mạng lưới thoát nước của thành phố Hồ Chí Minh, Công ty Thoát nước đô thị đã và đang triển khai xây dưng một hệ thống GIS nền desktop dựa trên phần mềm thưong mại ArcGIS và thu viện ArcEngine của ESRI. Trong hệ thống này, dũ liệu mạng luới thoát nước được tổ chức theo mô hình cơ sở dũ liệu đa người dùng Geodatabase và luu trũ trong một máy chủ đặt tại công ty. Để nhân viên của các xí nghiệp trực thuộc công ty có thể truy cập, khai thác tù xa thông tin $c o$ bản tù̀ co sở dũ liệu thoát nước này cần thiết phải xây dưng một hệ thống WebGIS. Với hệ thống WebGIS, nhân viên nếu được cấp quyền có thể truy cập đến cơ sở dũ liệu thoát nước tù moi nơi chỉ thông qua các trình duyệt web phổ biến. Để giảm bớt chi phi đầu tu, hệ thống WebGIS đã được xây dụng trên nền tảng các sản phẩm mã nguồn mở GeoServer và OpenLayers kết hợp sủ dụng ngôn ngũ lập trình Javascript và thu viện JQuery. Hệ thống cho phép thực hiện các hoạt động khai thác thông tin co bản nhu: i) hiển thị mạng luơoi thoát nước trên nền bản đồ Open Street Map; ii) lấy thông tin thuộc tính của đối tương thông qua tưong tác trục tiếp với đối tuợng trên màn hình; iii) thực hiện truy vấn tìm kiếm các đối tuợng dưa trên dũ liệu đa thuộc tính của chúng; iv) tìm kiếm các đối tượng trong mối quan hệ không gian với các đối tượng khác, ... Hệ thống đã được thử nghiệm và chưng tỏ tính hiệu quả trong khai thác co sở dũ liệu thoát nước qua internet.

\section{1. Đặt vấn đề}

Công ty Thoát nước đô thị Tp. Hồ Chí Minh đang xây dựng một hệ thống GIS nền Desktop để cho phép nhân viên của mình quản lý hiệu quả hệ thống thoát nước trên khu vực nội thành Tp. Hồ Chí Minh rộng hơn $400 \mathrm{~km}^{2}$. Trong hệ thống này, dữ liệu thoát nước được lưu trữ trong một máy chủ đặt tại khuôn viên của công ty. Công ty có một trụ sở chính và 10 xí nghiệp duy tu thoát nước nằm ở các vị trí khác nhau và thách thức là mỗi xí nghiệp duy tu phải được phép truy cập, sử dụng và cập nhật thông tin hạ tầng thoát nước thuộc phần xí nghiệp quản lý, trong khi trụ sở chính phải có khả năng quản lý toàn bộ mạng lưới và cơ sở dữ liệu thoát nước. Để giải quyết vấn đề truy cập dữ liệu từ xa đối với các xí nghiệp thoát nước, một giải pháp hiện đang được áp dụng là phát triển một mạng riêng ảo. Về cơ bản mạng riêng ảo (VPN) là một mạng cục bộ sử dụng hệ thống mạng công cộng sẵn có như internet để kết nối các văn phòng cũng như nhân viên ở xa. Với giải pháp này các xí nghiệp trực thuộc đã có thể truy cập đến cơ sở dữ liệu thoát nước sử dụng các phần mềm GIS. Nhưng khó khăn lại phát sinh là chi phí lớn cần có để trang bị số lượng lớn các phần mềm GIS cho các tác nghiệp hàng ngày tại các xí nghiệp trực thuộc. Hướng giải quyết cho vấn đề này là chỉ nên trang bị một số lượng đủ dùng các phần mềm GIS cho các công việc đòi hỏi phải sử dụng các chức năng phức tạp được thiết kế sẵn trong GIS, còn nếu người sử dụng chỉ cần truy cập xem thông tin bản đồ, xem thông tin thuộc tính hoặc thực hiện tìm kiếm các đối tượng thoát nước thì cần phải có một giải pháp khác. Một phải pháp có thể là phát triển một hệ WebGIS, trong đó dữ liệu và chức năng cần thiết được cung cấp thông qua internet. Người sử dụng của hệ thống thực hiện truy cập dữ liệu hoặc thực hiện các chức năng thông qua các giao diện thân thiện người dùng và đơn giản thông qua một trình duyệt web thông thường. Hai Map Server phổ biến đã được xem

Ngày nhận bài: 05/7/2019, ngày chuyển phản biện: 09/7/2019, ngày chấp nhận phản biện: 15/7/2019, ngày chấp nhận đăng: 18/7/2019 
xét: Minnesota MapServer [6], and GeoServer [3]. Mặc dù cả hai đều hỗ trợ OGC's Web Map Service (WMS) [8], MapServer chỉ hỗ trợ các yêu cầu cơ bản là xem và tra xét thông tin thông qua Web Feature Service (WFS), trong khi GeoServer thực hiện luôn cả các yêu cầu chèn, xóa và cập nhật dữ liệu. Công ty muốn người dùng của hệ thống có thể thực hiện một số hoạt động biên tập dữ liệu trên cơ sở dữ liệu trong tương lai, do vậy GeoServer đã được chọn như là một Map Server. Trước khi phát triển WebGIS, Công ty thoát nước đô thị cũng đã phát triển các ứng dụng web khác. Các ứng dụng Web này sử dụng Internet Information Services (IIS) của Microsoftnhư Web Server. Do vậy, WebGIS được xây dựng cũng sẽ sử dụng IIS Web Server. Như vậy, GeoServer, OpenLayers, và IIS Web Server được tích hợp để tạo khung nền tảng cho hệ thống WebGIS được xây dựng. Việc nghiên cứu ứng dụng WebGIS để khai thác thông tin thoát nước đã được giới thiệu trong [10], [11]. Tuy nhiên, cho đến thời gian gần đây, đã có một số nâng cấp về phía phần mềm, ví dụ Openlayers từ v.2 đã nâng cấp thành v.5.3, đã có một số công nghệ mới được giới thiệu do vậy bài báo này giới thiệu một số điều chỉnh và phân tích chi tiết hơn so với [10], [11].

\section{Thiết kế hệ thống WebGIS}

Hệ thống WebGIS đã được thiết kế với các thành phần như được mô tả dưới đây:

\subsection{Kiến trúc tổng quát}

Hệ thống WebGIS xây dựng được thiết kế theo mô hình kiến trúc 3 tầng như hình 1 trên cơ sở tham khảo tài liệu [1], [2] và các tài liệu khác:

- Tầng trình bày: đơn thuần là một trình duyệt Web, ví dụ Mozilla Firefox, để mở ứng dụng web có thiết kế định sẵn. Úng dụng Web được viết bằng ngôn ngữ Javascript, sử dụng kết hợp thư viện Jquery [4], thư viện mã nguồn mở Javascript OpenLayers v.5.3.0. OpenLayers được sử dụng để truy vấn và hiển thị thông tin bản đồ theo các chuẩn định dạng WMS/WFS. Úng dụng Web được phát triển để cung cấp các chức năng cơ bản thường gặp trong các phần mềm GIS desktop cơ bản. Thiết kế chi tiết chức năng của ứng dụng Web phía máy khách sẽ được thảo luận chi tiết sau.

- Tầng giao dịch: sử dụng Internet Information Services (IIS) Web Server tạo bởi Microsoft và phần mềm mã nguồn mở GeoServer có chức năng như một Map Server để cung cấp các dữ liệu và dịch vụ liên quan đến bản đồ theo các chuẩn dịch vụ WMS/WFS.

- Tầng dĩ liệu: Công ty thoát nước đã phát triển hệ thống Desktop GIS để quản lý dữ liệu của mạng lưới thoát nước. Những dữ liệu này được tổ chức theo mô hìnhcơ sở dữ liệu đa người dùng Geodatabase và lưu trong cơ sở dữ liệu quan hệ SQL Server 2008. Để đảm bảo tính toàn vẹn và nhất quán của hệ thống thông tin thoát nước, hệ WebGIS xây dựng sẽ chia sẻ và khai thác cơ sở dữ liệu đã thiết kế này.

\subsection{Co' sở dĩ liệu}

Dữ liệu mạng lưới thoát nước được lưu trữ theo hệ tọa độ VN2000 và theo mô hình cơ sở dữ liệu Geodatabase đa người dùng. Có hai nhóm dữ liệu: nền và chuyên đề.

Dữ liệu nền: Thể hiện các thực thể địa lý cơ bản (ranh giới, thủy hệ, giao thông,...) làm nền cơ sở về mặt không gian để nhận dạng và thể hiện vị trí các lớp dữ liệu chuyên đề thoát nước.

Dữ liệu chuyên đề: Dũ liệu chuyên ngành thoát nước, bao gồm: tuyến cống, hầm ga, trạm bơm, cửa xả,... (Xem hình 1)

\subsection{Chức năng cơ bản của hệ thống}

Do tính chất phức tạp của việc xây dựng ứng dụng trên Web, cũng như xuất phát từ yêu cầu xây dựng WebGIS để đáp ứng trước mắt các yêu cầu tra xét thông tin đơn giản, chỉ những chức năng đơn giản, thực sự cần thiết mới được thực hiện. Một số chức năng cơ bản đã được thực hiện sẽ được trình bày trong phạm vi bài báo này, bao gồm:

a) Hiển thị bản đồ: Cho phép chọn nạp và hiển thị các lớp dữ liệu nền Open Street Map $(O S M)$, chọn nạp và hiển thị các lớp dữ liệu 
chuyên đề thoát nước dưới dạng ảnh WMS hoặc dưới dạng đối tượng WFS; các lớp dữ liệu đã nạp có thể tắt/bật hiển thị hoặc có thể loại bỏ khỏi bản đồ khi cần thiết.

b) Tương tác với bản đồ: phóng to, thu nhỏ, rê bản đồ; truy cập ngược/xuôi các trang bản đồ đã xem trước đó; tạo, quản lý và di chuyển bản đồ nhanh đến các trang bản đồ được đánh dấu.

c) Tra xét, tìm kiếm thông tin: Hệ thống WebGIS đã xây dựng cho phép:

- Tra xét thông tin thuộc tính của các đối tượng thoát nước thông qua tương tác trực tiếp trên màn hình.

- Tra xét cơ sở dữ liệu nền của $O S M$ để tìm kiếm đối tượng dựa trên địa chỉ của đối tượng. Khi đối tượng được tìm thấy, vị trí của đối tượng được thể hiện trên bản đồ.

- Tra xét cơ sở dữ liệu chuyên đề thoát nước dựa trên nhiều điều kiện về thuộc tính của đối tượng. Các đối tượng tìm thấy được đánh dấu trên bản đồ.

- Tìm kiếm các đối tượng thoát nước trong mối quan hệ không gian với các đối tượng thoát nước khác.

\subsection{An toàn của hệ thống}

Hệ thống WebGIS được xây dựng không hỗ trợ quản lý, tạo mới người dùng qua Internet mà chỉ thực hiện việc kiểm tra, xác thực người dùng thông qua tên và mật khẩu của người dùng hiện đang sử dụng hệ thống GIS nền desktop. Trường hợp người dùng không được xác thực thì không thể truy cập đến nội dung bản đồ của trang WebGIS đã xây dựng.

\subsection{Giao diện của trang WebGIS}

Hình 2 thể hiện thiết kế của giao diện chính của trang WebGIS. Trong giao diện này, mỗi chức năng của hệ thống sẽ được gọi thực thi thông qua một hoặc một vài công cụ. Các công cụ được thể hiện dưới dạng các icons trên màn hình và được bố trí nằm ở phần đỉnh của màn hình. Nằm về phía đáy của màn hình là dòng trạng thái dùng thể hiện thông tin về tọa độ của con trỏ, tỉ lệ của bản đồ và các thông báo (nếu có) liên quan đến kết quả xử lý dữ liệu. Toàn bộ màn hình dùng thể hiện bản đồ. Tất cả những cửa sổ hoặc những bảng thoại xuất hiện trong quá trình tương tác với trang Web đều ở dạng cửa sổ nỗi và sẽ biến mất sau khi xử lý xong.

\subsection{Ngôn ngữ lập trình}

Các tương tác phía ứng dụng được viết sử dụng Hypertext Markup Language (HTML) và Javascript. Tương tác với Server cũng được viết bằng Javascript với sự hỗ trợcủa Node.js [7] một nền tảng chạy trên môi trường V8 Javascript Runtime. (Xem hinh 2)

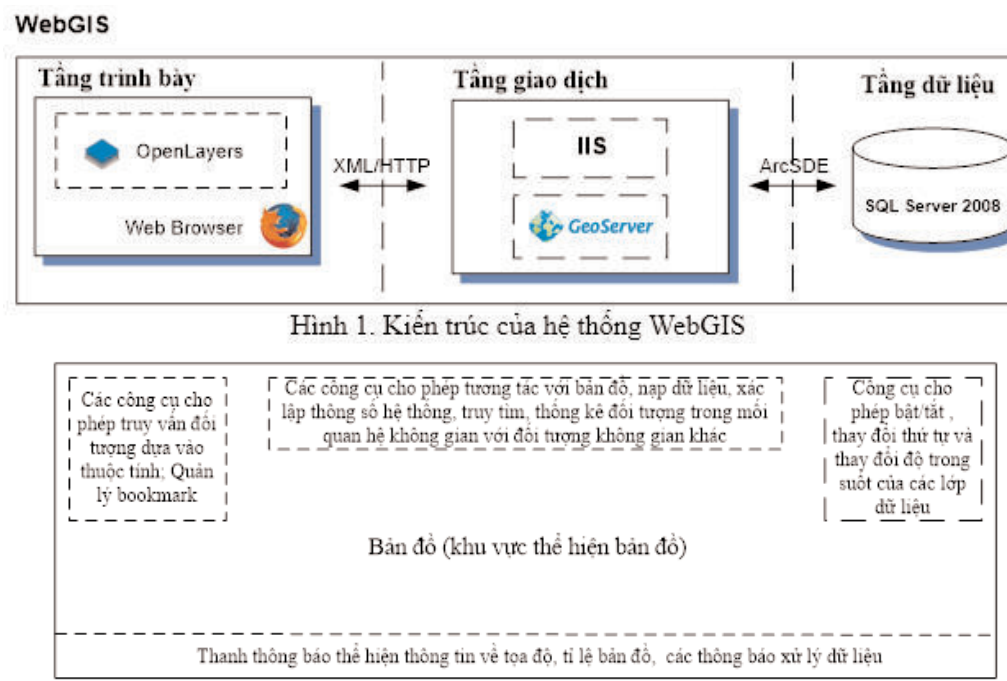

Hình 2. Bố trí các phần giao diện của trang WebGIS 


\section{Phát triển hệ thống WebGIS}

Hệ thống WebGIS được phát triển trên cơ sở những phân tích, thiết kế ở trên. Nội dung tiếp theo, giải thích các API nào trong thư viện OpenLayers 5.3.0 đã được sử dụng để xây dựng nên một số chức năng đã thực hiện.

\subsection{Hiển thị bản đồ}

Một bản đồ Web phía client được tạo ra với việc sử dụng API ol.Map từ thư viện OpenLayers. Lớp dữ liệu nền $O S M$ được tạo và hiển thị như là tập hợp các tấm ảnh bản đồol.layer.Tile với nguồn dữ liệu xác định là ol.source.OSM. Các lớp dữ liệu chuyên đề thoát nước có thể được tạo dưới dạng WMS hoặc WFS. Các lớp dữ liệu WMS được tạo - cũng giống như lớp OSM - như là tập hợp các tấm ảnh bản đồol.layer.Tile tuy nhiên với nguồn dữ liệu được yêu cầu hoặc ol.source.ImageWMS hoặc ol.source.TileWMS. Các tham số đi kèm với các yêu cầu về cơ bản là địa chỉ URL của Map Server, tên của lớp dữ liệu yêu cầu cùng với khu vực địa lý quan tâm. Các lớp dữ liệu WFS được tạo bằng cách gọi thực thi yêu cầu ol.layer.Vector với nguồn dữ liệu xác định là ol.source.Vector, kèm yêu cầu cung cấp một dịch vụ WFS GetFeature đối với Map Server. Kết quả trả trở về dưới dạng một tập hợp các đối tượng với đầy đủ dữ liệu thuộc tính và tọa độ hình thành nên đối tượng. Tại máy khách, thư viện OpenLayers sẽ chịu trách nhiệm thể hiện các đối tượng này dưới dạng bản đồ. Để có thể hiển thị được trên bản đồ, các lớp dữ liệu cần phải được nạp vào bản đồ sử dụng hàm của map là addlayer().

Số lượng và dung lượng bộ nhớ tạo ra cho mỗi lớp dữ liệu là rất lớn. Việc truyền dữ liệu qua lại giữa máy chủ và máy khách sẽ mất nhiều thời gian, do vậy ứng dụng được thiết kế sao các lớp dữ liệu thoát nước sẽ chỉ được tạo và thể hiện khi người sử dụng có yêu cầu. Danh sách các lớp dữ liệu được cung cấp nhờ vào việc gọi yêu cầu getCapabilities đối với Map Server.

\subsection{Tương tác với bản đồ}

Tương tác với bản đồ có thể được thực hiện thông qua các controls tạo sẵn bởi thư viện OpenLayers. Các controls này cho phép người sử dụng có thể tương tác với bản đồ, ví dụ như rê bản đồ, phóng to, thu nhỏ, xem toàn cảnh,... Để sử dụng được các controls, chúng phải được thêm vào bản đồ thông qua ol.map.addControl(). Tùy mục đích, người sử dụng có thể tùy chọn các controls phù hợp để đưa vào sử dụng. Một số controls có sẵn trong thư viện OpenLayers đã được khai thác sử dụng trong hệ thống WebGIS này: ol.control.MousePosition để nhận vị trí pointer trên màn hình; ol.control.ZoomSlider để phóng to/thu nhỏ bản đồ; ol.control.OverviewMap để nhận được toàn cảnh bản đồ; ol.control.ScaleLine để nhận thông tin về tỉ lệ bản đồ.

Hình 3 minh họa giao diện hoàn chỉnh của hệ thống, với các controls đã được xây dựng cùng với dữ liệu đoạn cống và hố ga nằm chồng lên trên bản đồ nền $O S M$ của khu vực nghiên cứu.

\subsection{Tra xét, tìm kiếm thông tin}

a. Tra xét thông tin thuộc tính của đối tượng thoát nước thông qua tương tác trực tiếp với đối tượng trên bản đồ: Công việc này được thực hiện

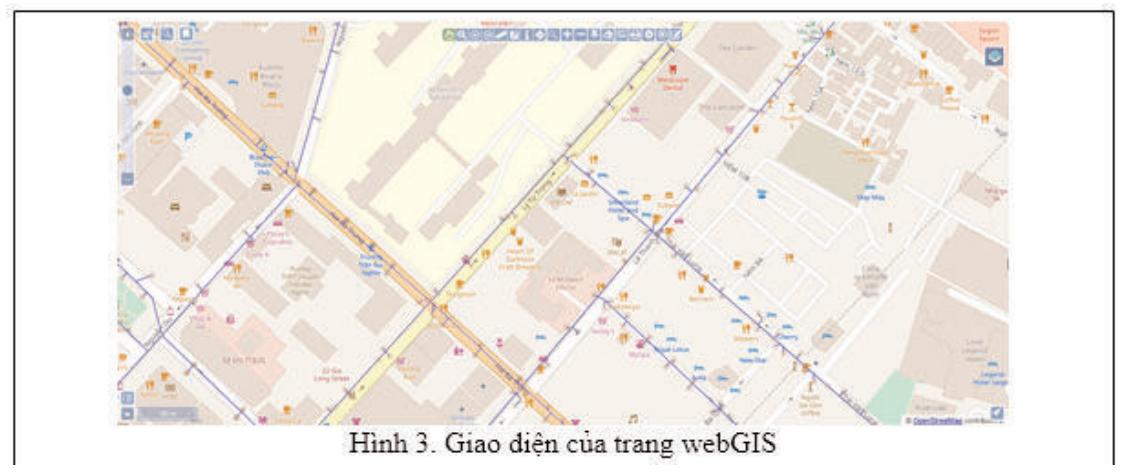


với việc sử dụng control chuyên biệt được phát triển thêm. Tùy thuộc vào điều kiện lớp dữ liệu cần tra xét thông tin là thuộc loại WMS hoặc WFS, mà cách thức tra xét thông tin sẽ khác nhau. Nếu lớp dữ liệu là WMS, một yêu cầu $X M L H t t p R e q u e s t G E T$ được gửi đến Map Server với các thông số như địa chỉ Server, dịch vụ GetFeature, tên lớp dữ liệu được yêu cầu cùng với việc đưa vào điều kiện hình học intersects (giao) giữa các đối tượng trong lớp dữ liệu với hình vuông bao xung quanh điểm tìm kiếm $(1 / 2$ cạnh của hình vuông có giá trị mặc định là 5 pixel). Còn nếu lớp dữ liệu là WFS, do các đối tượng tìm kiếm đã được nạp về máy khách, hàm forEachFeatureAtPixel của ol.map sẽ được thực thi ngay tại máy khách để tra xét tìm kiếm các đối tượng có hình thể giao với hình chũ nhật bao xung quanh điểm tìm kiếm. Khi đối tượng được tìm thấy, thuộc tính của đối tượng sẽ được đưa vào popupwindow và được hiển thị như hình 4 :

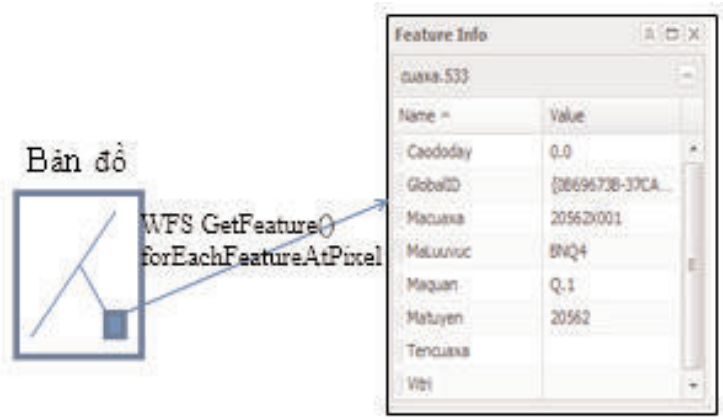

Hình 4: Tra xét trục tiếp thuộc tính đối tượng trên bản đò̀

b. Tìm kiếm đối tượng thoát nước dựa trên thuộc tính của chúng: giống như trong trường hợp trên phải chia ra trường hợp lớp dữ liệu là WMS hoặc WFS. Nếu lớp dữ liệu là WMS, một yêu cầu XMLHttpRequest GET hoặc POST được gửi đến Map Server với các thông số như địa chỉ server, dịch vụ GetFeature, tên lớp dữ liệu được yêu cầu cùng với việc đưa vào các điều kiện lọc, có thể sử dụng <ogc:Filter>. Nếu có đối tượng được tìm thấy, thì các đối tượng được thể hiện trên màn hình và bản đồ sẽ phóng đại và di chuyển đến khu vực chứa các đối tượng được tìm thấy. Ví dụ, để tìm kiếm đối tượng cửa xả thuộc lưu vực Bắc Thành phố Hồ Chí Minh một yêu cầu XMLHttpRequest GET được gửi với dữ liệu như sau:

http://localhost:8081/GeoServer/wfs?request $=$ GetFeature \&version $=1.1 .0 \&$ service $=$ wfs \& type Name=thoatnuocwksp:cuaxa\&CQL_FIL$\mathrm{TER}=$ MaLuuvuc $=$ 'BTP'.

Trong trường hợp lớp dữ liệu là WFS, từng đối tượng tại máy khách sẽ được kiểm tra thuộc tính xem có thỏa mãn điều kiện tìm kiếm hay không. Các đối tượng thỏa mãn điều kiện sẽ được đánh dấu và màn hình sẽ phóng đại và di chuyển đến khu vực chứa các đối tượng được tìm thấy.

Để hỗ trợ cho việc tìm kiếm đối tượng dựa vào thuộc tính, một giao diện tìm kiếm được cung cấp giống như hình 5 , cho phép người sử dụng tìm kiếm đối tượng thuộc một lớp dữ liệu đã chọn, tìm kiếm dựa trên số lượng các điều kiện thuộc tính do người sử dụng quyết định, ví dụ trên hình 5 chọn tìm dựa theo 2 điều kiện "Maluuvuc $=$ BTP và Kichthuoc $=800 "$ ". Nếu muốn thêm điều kiện thì nhấn chọn " $A d d$ rule".Khi đối tượng được tìm thấy đối tượng được đánh dấu, và bản đồ sẽ được phóng đại và dịch chuyển đến khu vực chứa đối tượng tìm thấy.

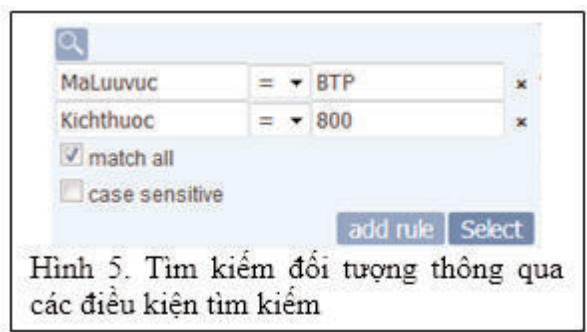

c. Hệ thống WebGIS đã xây dựng cũng cho phép tra xét thông tin nền của lớp dữ liệu OSM. Để tìm kiếm nhấn vào control, gõ vào ô thông tin địa chỉ cần tìm kiếm, ví dụ để tìm trường Đại học Bách Khoa Tp. HCM có thể gõ "268, Ly Thuong Kiet”. Một yêu cầu được gởi đến server của OSM, có dạng https://nominatim.openstreetmap.org $/ \mathrm{search}$ ? format $=j$ son \&addressdetails $=1 \& q=268 \% 2 \mathrm{C} \% 20 \mathrm{Ly} \% 20 \mathrm{Thuong} \% 20 \mathrm{Kie}$ 
$\underline{t \& p o l y g o n ~ g e o j s o n=1 \& \text { limit }=10}$. Nếu có dũ liệu địa chỉ tương tự với địa chỉ cần tìm, dữ liệu sẽ được trả trở về từ Server - với thông tin địa chỉ và thông tin hình thể - và được thể hiện như một danh sách cho phép người sử dụng chọn lựa

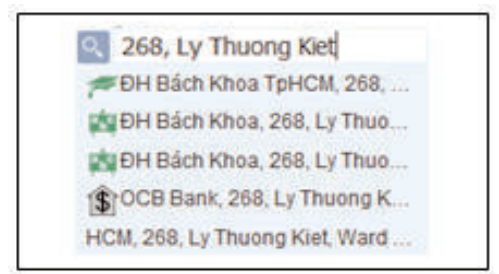

Sau khi người sử dụng đã chọn lựa một dòng thích hợp trong danh sách, màn hình sẽ dịch chuyển đến vị trí đối tượng được tìm thấy.

d. Hệ thống WebGIS đã xây dựng cũng cho phép tìm kiếm đối tượng thoát nước trong mối quan hệ không gian với một đối tượng không gian xác định. Để hỗ trợ cho việc tìm kiếm, một giao diện tìm kiếm được cung cấp giống như hình 6 .

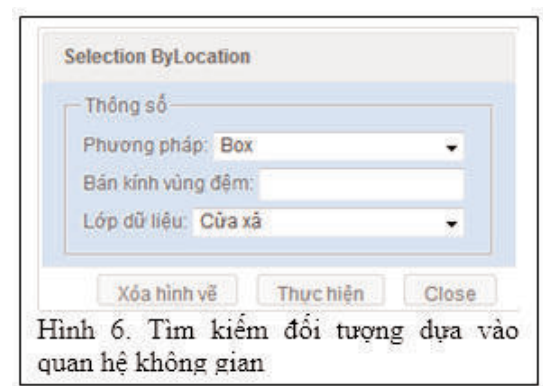

Cách thức thực hiện bao gồm: i) một đối tuoọng không gian xác định sẽ được xác định bằng 1 trong nhiều phương pháp, có thể vẽ một hình chữ nhật (Box), một hình tròn,..., hoặc bằng cách chọn một đối tượng thoát nước có sằn; ii) cung cấp một giá trị thể hiện bán kính vùng đệm; và iii) xác định lớp dữ liệu có đối tượng cần tìm kiếm giao với đối tượng xác định. Khi nhấn nút thực hiện, một yêu cầu getFeature() sẽ được gửi về Server nếu đối tượng thuộc lớp WMS cùng với nó là một yêu cầu lọc các đối tượng trong lớp dữ liệu có hình thể intersects (giao) với hình thể của đối tượng xác định đã định ở bước i) hoặc giao với vùng đệm xung quanh đối tượng xác định với bán kính đã chọn. Các đối tượng tìm thấy sẽ được đánh dấu và thể hiện trên bản đồ. Trong trường hợp các đối tượng cần tìm thuộc lớp dữ liệu WFS, quan hệ không gian giữa các đối tượng được xác định bằng cách sử dụng hàm giao intersects của thư viện JSTS[5] và sẽ được thực hiện ngay tại máy khách. Chú ý, thư viện này cần phải được khai báo địa chỉ và nạp trước vào trang Web tại máy khách.

\section{Kết luận}

Tích hợp GIS và công nghệ Web đã mở ra rất nhiều cơ hội cho việc chia sẻ thông tin thông qua internet. Bài báo này giới thiệu cơ hội đã được vận dụng như thế nào trong việc khai thác dữ liệu không gian về mạng lưới thoát nước thông qua mạng internet. Chú trọng đến việc giảm chi phí đầu tư, hệ thống được xây dựng bằng cách sử dụng các sản phẩm phần mềm mã nguồn mở như GeoServer và OpenLayers. Hệ thống được tiến hành thử nghiệm thành công tại công ty thoát nước đô thị thành phố Hồ Chí Minh. Với hệ thống này, nhân viên từ các xí nghiệp trực thuộc công ty thoát nước đô thị không cần phải có một phần mềm GIS mà chỉ cần có một trình duyệt Web và Internet là đã có thể truy xuất tới cơ sở dữ liệu thoát nước lưu trữ tại trụ sở chính của công ty, xem và tra xét thông tin mạng lưới thoát nước một cách dễ dàng. Mặc dầu hệ thống WebGIS đã xây dựng cho phép người sử dụng linh động trong việc chọn làm việc với các lớp dữ liệu chuyên đề thoát nước dưới hai dạng WMS và WFS, nếu mục đích của việc sử dụng chỉ là xem hoặc tra xét thông tin với các đối tượng thoát nước có số lượng lớn - ví dụ lớp hố ga có hàng trăm ngàn đối tượng với hàng chục cột thuộc tính - tốt nhất nên làm việc với dữ liệu này ở dạng WMS để giảm thời gian phải chờ đợi do dữ liệu chi tiết - thuộc tính và hình thể - của tất cả các đối tượng cần phải được chuyển từ máy chủ về máy khách trước khi hoạt động hiển thị hoặc bất kỳ hoạt động xử lý nào có thể tiến hành. $O$

\section{Tài liệu tham khảo}

[1]. AA. Alesheikh, H. Helali, HA.Behroz, Web GIS, Technologies and Its Applications, Symposium on Geospatial Theory, Processing 
and Applications, Ottawa, 2002.

[2]. Zhong Ren Peng and Ming Hsiang Tso, Internet GIS.John Wiley and Sons, Inc, 2003.

[3]. Geoserver, http://www.geoserver.org/

[4]. JQuery, https://jquery.com/

[5]. JTS Topology https://github.com/locationtech/jts

[6]. MapServer, http://mapserver.org

[7]. Node.js, https://nodejs.org/en/

[8]. Open Geospatial Consortium Inc., OpenGIS Web Processing Service, Version 1.0.0, Document number OGC 05-007r7, 2007.
[9]. OpenLayer

User

Guide, http://www.openlayers.org

[10]. Trần Trọng Đức, Developing a WebGIS application to exploit drainage network data via the internet,International Conference on GeoInformatics for Spatial-Infrastructure Development in Earth \& Allied Sciences, 2016

[11]. Trần Trọng Đức, Integration of Geoserver, OpenLayer, GeoExt and ArcSDE to manage an Urban Drainage Information via Internet, Int. Sym. on GeoInformatics for Spatial-InfraStructure Development in Earth and allied sciences, Hà Nội - Việt Nam, 2010.O

\section{Summary}

\section{Development of WebGIS system to exploit drainage-network data via the internet}

Tran Trong Duc

\section{Ho Chi Minh city University of Technology, VNU - HCM}

To manage drainage-network system of Hochiminh city, the city's Urban Drainage Company has been developing a desktop GIS based on ArcGIS software, and ArcEngine library of ESRI. In this system, drainage database is organized into Geodatabase model and is stored in a server placed at the company headquarter. In order to allow staffes from the company's satellite offices get access to the server's database, it is necessary to build a WebGIS system. With this webGIS, the staff with permission can access to drainage database from everywhere through the Internet by using just a normal web browser. To reduce investment cost, a WebGIS system has been developed based on open source products Geoserver, and OpenLayerscombined using programming language Javascript and Jquery library. The system allows to implement basic functionssuch as i) display drainage-network data on base map of Open Street Map ii) retrieve attributes by direct interact with drainage features on map iii) search for features based on their attributes iv) search for features in spatial relationship with other features, ... The system has been tested and proved to work effectively in exploiting the drainage-network database through the internet. $\bigcirc$ 